\title{
Temperature-dependent modulated reflectance of InAs/lnGaAs/GaAs quantum dots-in-a-well infrared photodetectors
}

\author{
R. Nedzinskas, ${ }^{1, a)}$ B. Čechavičius, ${ }^{1}$ A. Rimkus, ${ }^{1}$ E. Pozingytè,${ }^{1}$ J. Kavaliauskas, ${ }^{1}$ \\ G. Valušis, ${ }^{1}$ L. H. Li, ${ }^{2}$ and E. H. Linfield ${ }^{2}$ \\ ${ }^{1}$ Semiconductor Physics Institute, Center for Physical Sciences and Technology, A. Goštauto 11, LT-01108 \\ Vilnius, Lithuania \\ ${ }^{2}$ School of Electronic and Electrical Engineering, University of Leeds, Leeds LS2 9JT, United Kingdom
}

(Received 26 January 2015; accepted 28 March 2015; published online 10 April 2015)

\begin{abstract}
We present a photoreflectance (PR) study of multi-layer InAs quantum dot (QD) photodetector structures, incorporating InGaAs overgrown layers and positioned asymmetrically within GaAs/AlAs quantum wells (QWs). The influence of the back-surface reflections on the QD PR spectra is explained and a temperature-dependent photomodulation mechanism is discussed. The optical interband transitions originating from the QD/QW ground- and excited-states are revealed and their temperature behaviour in the range of $3-300 \mathrm{~K}$ is established. In particular, we estimated the activation energy ( $\sim 320 \mathrm{meV})$ of exciton thermal escape from QD to QW bound-states at high temperatures. Furthermore, from the obtained Varshni parameters, a strain-driven partial decomposition of the InGaAs cap layer is determined. (C) 2015 AIP Publishing LLC.
\end{abstract}

[http://dx.doi.org/10.1063/1.4917204]

\section{INTRODUCTION}

Owing to their unique atomic-like quantum confinement, self-assembled semiconductor quantum dots (QDs) are particularly attractive from a fundamental research perspective and also for their industrial applications in optoelectronics, such as the development of QD telecom lasers ${ }^{1,2}$ and infrared photodetectors (QDIPs). ${ }^{3,4}$ Extensive experimental and theoretical studies have been performed on InAs QDs within GaAs matrix. More recently, QDIP structures have been implemented by covering the self-assembled InAs/GaAs QDs with a strain-relieving InGaAs cap layer. ${ }^{4}$ This dots-ina-well (DWELL) photodetector design is then based on intraband optical transitions between QD and quantum well (QW) bound-states and, hence, offers the additional possibility of engineering the detection peak wavelength by adjusting QW width and/or composition. Moreover, such QDIP schemes allow a bias-tunable spectrally adaptive response. Very recently, DWELL structures have been further modified by embedding InAs QDs within InGaAs/GaAs/ AlGaAs double wells, resulting in more efficient device operation due to a significant reduction in the dark current. ${ }^{5-7}$

Understanding the temperature dependence of the optical properties and electronic structure of DWELL devices is crucial for their optimization and further development. This can be obtained by photoluminescence (PL) and modulated reflectance spectroscopy, in particular, by photoreflectance (PR), phototransmittance (PT), and contactless-electroreflectance (CER) techniques. To date, these methods have been successfully applied to characterize a number of low-dimensional systems and nanostructures. ${ }^{8-17}$ But despite the previous extensive studies of quantum heterostructures, the temperature-dependent modulated reflectance spectra of multi-layer DWELL

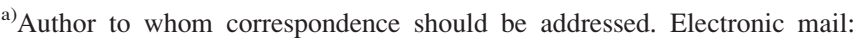
ramunas@pfi.lt
}

photodetector structures with doped QDs have yet to be investigated extensively, and further work is still needed to clarify the underlying mechanisms.

In this work, we perform in-depth spectroscopic study of multi-layer InAs QD structures, with InGaAs strainrelieving layers, situated asymmetrically inside GaAs/AlAs QWs. For the first time, we provide evidence for the influence of back-surface reflection (BSR) effects on the PR spectra of DWELL structures. Furthermore, we have carried out temperature-dependent PR and PL measurements, probing the QD- and QW-related interband optical transitions over the temperature range of $3-300 \mathrm{~K}$. A comparison of experimental results and numerical calculations of the electron energy structure provides a deeper insight into the electronic properties of DWELL structures.

\section{EXPERIMENTAL DETAILS: SAMPLES AND SETUP}

Our quantum heterostructures were designed as lateral transport photodetectors and were grown by molecular beam epitaxy on semi-insulating (100) GaAs substrates. DWELL samples then comprise a $400 \mathrm{~nm}$-thick undoped GaAs buffer layer, an absorbing 10 period InAs/InGaAs/GaAs/AlAs multiple heterostructure, and an undoped $80 \mathrm{~nm}$-thick GaAs top layer (see Fig. 1). Each period in the asymmetric active region consists of a $5 \mathrm{~nm}$-thick AlAs barrier layer followed by a $10 \mathrm{~nm}$-thick GaAs bottom-QW layer, a self-assembling InAs QD (2.4 ML) layer, a $5 \mathrm{~nm}$-thick strain-relieving $\mathrm{In}_{0.15} \mathrm{Ga}_{0.85} \mathrm{As} \mathrm{QW}$ layer, and a $20 \mathrm{~nm}$-thick GaAs top-QW layer. It was estimated, using atomic force microscopy on similar samples grown without the GaAs top layer, that the nominal height of the lens-shaped QD is approximately $5 \mathrm{~nm}$, the average dot diameter is about $20 \mathrm{~nm}$, and the sheet density of dots is $2 \times 10^{10} \mathrm{~cm}^{-2}$. Furthermore, the QDs were directly $\mathrm{Si}$-doped at a level corresponding to about one electron per dot. It should be noted that in our DWELL structures 


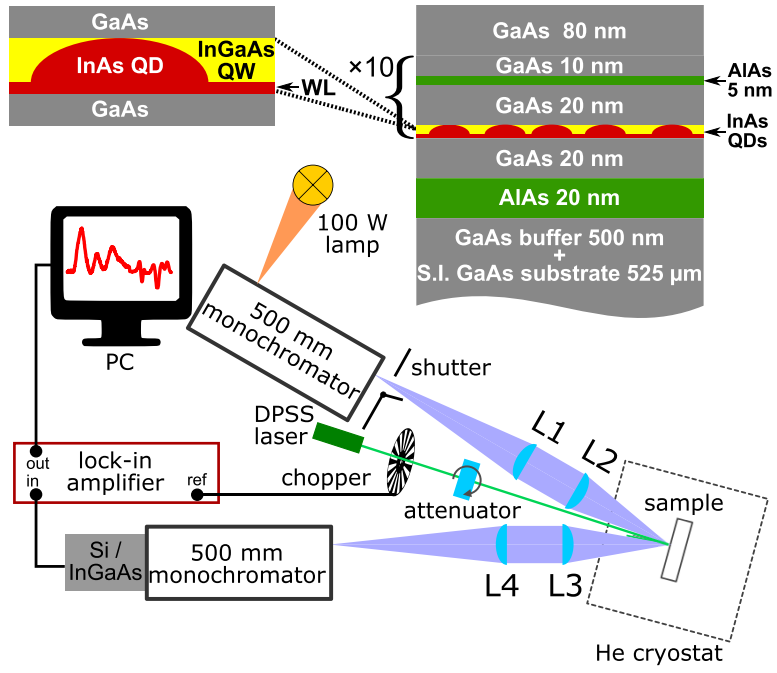

FIG. 1. Sketch of the DWELL structures studied (top), and the experimental setup for PR/PL spectroscopy using a dual-monochromator system.

an uncorrelated distribution of QDs is characteristic owing to the comparatively thick $(>20 \mathrm{~nm})$ separation between adjacent QD layers. Therefore, the separate InAs QD layers can be considered as electronically uncoupled.

The spectroscopic measurements were carried out in the temperature range of $3-300 \mathrm{~K}$ by mounting the samples on the cold finger of a closed-cycle helium cryostat. A diodepumped solid-state laser emitting a wavelength of $355 \mathrm{~nm}$ or $532 \mathrm{~nm}$ was used as the modulation source in PR/PT, and as the excitation source in PL. Laser light was modulated by a mechanical chopper $(\sim 270 \mathrm{~Hz})$, whilst the laser power density (intensity) was varied using neutral density filters and/or an attenuator (Rochon polarizer).

The PR/PT spectra were measured using a doublemonochromator system, as depicted in Fig. 1, which provided better signal-to-noise ratios under measurement conditions (compared to the conventional use of a low-pass filter), and allowed simultaneous collection of the PL spectrum. ${ }^{18}$ In this configuration, the probe source was a $100 \mathrm{~W}$ tungsten-halogen lamp, which was coupled into a $500 \mathrm{~mm}$ focal length monochromator (Andor SR-500i). The resulting quasi-monochromatic probe light was focused onto the sample, under nearly normal incidence, to a spot size of 2-3 mm in diameter, where it was overlapped by the laser pump beam. The probe light reflected from the sample was then collected into the second $500 \mathrm{~mm}$ monochromator, which synchronously followed the first. Consequently, this second monochromator behaved as an extremely selective band-pass filter. One should note that at lower temperatures the PR signal also contains a PL component so that $\Delta R+\mathrm{PL}$ is the actual quantity measured. Therefore, the $\Delta R / R$ (the true PR) spectrum was recovered by first subtracting the PL from the measured signal $(\Delta R+\mathrm{PL})$ and then normalizing it to $R$. PL spectra in this setup were separately taken by blocking the probe beam using a shutter.

Depending on the spectral range of interest, PR and PL signals were recorded either with $\mathrm{Si}$ or thermoelectrically cooled InGaAs photodetectors, which were attached to the exit slit of the second monochromator. Room temperature CER experiments were carried out using a capacitor-like system. ${ }^{9,14,16}$ To provide a modulating electrical field, a sinetype AC signal from a high voltage amplifier (Trek 609E-6) of $\sim 1.5 \mathrm{kV}$ peak-to-peak amplitude and $\sim 190 \mathrm{~Hz}$ frequency was applied to the top transparent electrode. A phasesensitive detection technique, with a lock-in amplifier, was used in all spectroscopic measurements.

\section{EXPERIMENTAL RESULTS AND DISCUSSION}

\section{A. Effects of the back-surface reflections}

Before presenting the temperature-dependent spectroscopic data, let us consider the role of BSR on modulated reflectance spectra in more detail. Previously, the BSR effect on PR spectra has been studied for GaAs, ${ }^{19,20}$ but not for QD structures. The probability of an absorption process occurring in the QD layer is defined by the absorption crosssection, $\sigma=\alpha / N$, where $\alpha$ is absorption coefficient and $N$ is the QD sheet density. One should take into account that due to a low $\sigma$ and limited number of QD layers, the probing light penetration depth $(1 / \alpha)$ at photon energies below the fundamental absorption edge of GaAs, $\hbar \omega<E_{\mathrm{g}}^{\mathrm{GaAs}}$, exceeds the total thickness of the DWELL structure (including the undoped GaAs substrate). Under these circumstances, for asgrown samples with specular back-surfaces, the effect of BSR can become significant.

In order to compare PR spectra directly, with and without light reflected from the back-surface, we cut the DWELL samples into two pieces and roughened the bottom specular surface (substrate) of one piece with sandpaper. We then experimentally investigated the BSR effect in DWELL structures by measuring the PR (see Fig. 2) along with unmodulated reflectance $R$ and transmittance $T$ (see Fig. 3) spectra of as-grown and sanded samples. In Fig. 2, we also included PT, CER, and high-excitation PL spectra for comparison.

Three major sets of spectral features, related to various interband transitions in the DWELL structure, can be identified in optical spectra measured at room temperature and depicted in Fig. 2. Strong high energy features above the bandgap of GaAs $(1.42 \mathrm{eV})$ are ascribed to interband transitions in the wide GaAs/AlAs QW, which are superimposed by Franz-Keldysh oscillations from the GaAs cap layer. In the photon energy range between 1.25 and $1.42 \mathrm{eV}$, several sharp PR features correspond to optical transitions in the InAs/InGaAs binary quantum well (bi-QW), formed by the InAs wetting layer (WL) and InGaAs cap. In the low photon energy range of $0.9-1.25 \mathrm{eV}, \mathrm{PR}$ and PL spectra exhibit several broadened features due to ground-state (GS) and excited-state (ES) interband transitions of the QD ensemble. The origin of these QD-related features is confirmed by the high-excitation PL spectrum. The prominent multiple peaks in the PL spectrum signify that the QDs are quite uniform layer-by-layer. Hereinafter, we will primarily focus on the pronounced differences in the line shape of the QD photomodulated spectra.

As is evident from Fig. 2, the intensity and line shape of QD features in the photomodulated spectrum for as-grown samples match reasonably with $\mathrm{PT}$, but are completely 


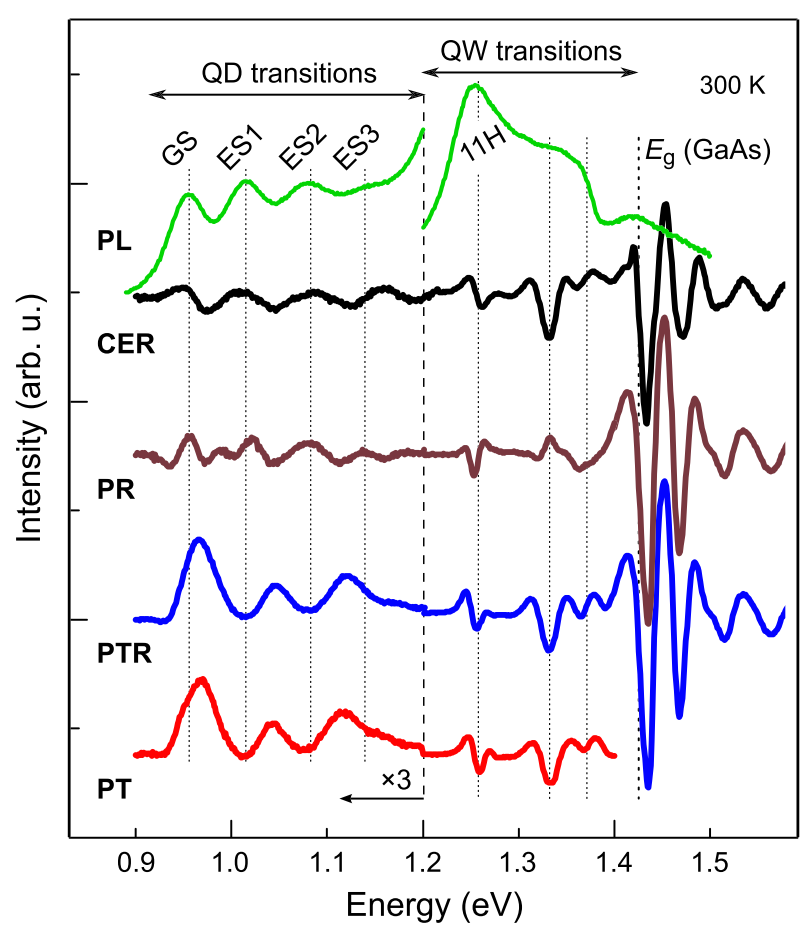

FIG. 2. Comparison of high-excitation photoluminescence (PL), contactless electroreflectance (CER), photoreflectance (PR), phototransmittance in a reflection geometry (PTR) and phototransmittance (PT) spectra for the DWELL structure measured at room temperature. PR data were taken on the roughened sample.

different from the PR features of roughened samples. This can be explained by considering BSR effects. Indeed, as can be seen in Fig. 3, the featureless reflectivity spectrum for asgrown (specular) samples in the photon energy range just below the bandgap of GaAs exhibits a sharp step, which disappears after sanding the rear surface of the sample. The reflectivity step is perfectly matched with the edge of the transmittance spectrum (see Fig. 3) and evidently appears due to multiple reflection effects between front and back surfaces of the sample.

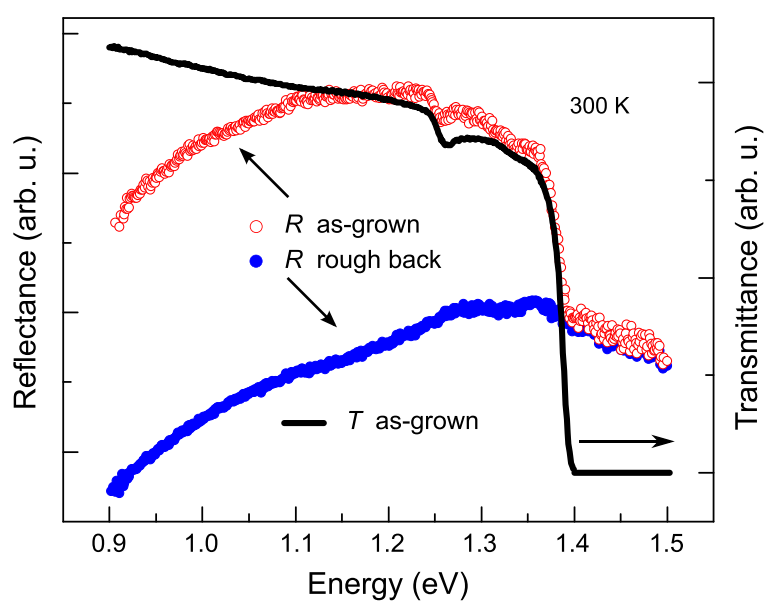

FIG. 3. Room temperature transmittance $(T)$ and reflectance $(R)$ spectra of the DWELL structure for the sample with specular (as-grown) and rough back-surfaces. The rough back-surface scatters the probing light and eliminates the BSR component.
These results support the presumption that the photomodulated signal for as-grown QD structures with a mirror-like back-surface is actually a superposition of two components. The first one is associated with the field-induced change of the light reflected at the front-side interface and is commonly known as the PR. This "proper" PR is distinguished in the photomodulated spectra of QD structures with rough backsurfaces (see PR spectrum in Fig. 2). The second component, referred to as PT in reflection mode (PTR), is related to the light partly reflected at the back-side interface. Likewise in a traditional PT experiment, the PTR components emerge due to the photoabsorption (PA) effect. ${ }^{20}$ Indeed, the PTR spectrum is very similar to the PT spectrum in the low photon energy range of QD-related optical transitions, as shown in Fig. 2. It can thus be seen that PA effect dominates in the PTR spectrum of QDs for as-grown samples.

The predominance of the PA effect in the modulated reflectance spectrum of InAs QDs can be explained by considering light traveling inside the as-grown sample. The back-reflected light passes twice (forth and back) through all the QD layers and consequently is the product of the PA of every individual QD layer. Neglecting the small multiple reflections between QD layers, PTR measures the quantity $\Delta T / T \approx-2 \Delta \alpha d$, where $d$ is the thickness of QD layers producing the signal. On the other hand, the PR signal depends only weakly on the number of QD layers, in analogy with previous experiments on multiple-QWs. Instead, increasing the number of QW layers only changes the phase of the line shape. $^{21}$ Note, however, that the QD structures under study are not strictly periodic because the QDs are not vertically aligned and their in-plane positions are inherently random. It also should be noted from Fig. 2 that the BSR effect is insignificant in the CER spectrum. We speculate that this phenomenon is associated with the short modulation depth in CER spectroscopy. ${ }^{16}$

\section{B. Photomodulation mechanisms}

As it is evident from Fig. 2, the lineshape symmetry of QD features in PT and PTR spectra is quite different from those in PR spectra. In particular, in the region of GS transitions, PT and PTR clearly show positive signals (induced transmission), while PR and CER spectra exhibit a dispersive-like shape. By analyzing these data, we can discern two modulation mechanisms that contribute to the absorption change: (i) state-filling and (ii) the quantumconfined Stark effect (QCSE). For the studied heterostructures, parts of the QD layers are located in the space-charge region formed near the surface (as well as from the buffer layer) and consequently these QDs are subjected to relatively strong electric fields, preventing carrier occupation within dots. Thanks to a short penetration depth of the modulating laser beam used in the experiment, ${ }^{22}$ the PR signal from the topmost QD layers is dominated by QCSE, associated with screening of the surface electrical field due to (laser) generated free electron-hole pairs. Such a photomodulation mechanism was predicted and is widely accepted for undoped QDs. ${ }^{10}$ On the other hand, the photomodulated signal which originates from the QD layers located outside the surface 
space-charge region can be interpreted in terms of statefilling mechanism. This mechanism accounts for the Pauli blocking of interband transitions in the QDs and Coulomb carrier-carrier interaction effects. ${ }^{23}$ It can be assumed that both of the aforementioned effects govern PT line shapes of QD-related optical transitions. According to the Pauli principle, electron occupation of QD states suppresses the absorption, and this is manifested as positive peaks in PT and PTR spectra of the GS transitions. Coulomb interactions shift mainly the unpopulated ESs, as is seen by their dispersive spectral form.

The optical transitions in QD structures responsible for the observed spectral features were examined on the basis of line shape analysis of the modulated reflectance spectra. The model used in the analysis is based on the fact that both state-filling and the QCSE are expected to influence the photomodulated spectra. In accordance with Refs. 24 and 25, the QDs' state-filling signal is proportional to the number of modulated electrons due to the Pauli blocking effect. Its line shape will be a first derivative of the dielectric function with respect to the intensity or oscillator strength, and can be described by a Gaussian function. On the other hand, the Stark shift can be represented by the derivative of the Gaussian function.

To determine the optical transition energies and broadening parameters from the recorded optical features, modulated reflectance spectra were analyzed through least squares fitting using a Lorentzian-type function ${ }^{26}$

$$
\Delta R / R=\operatorname{Re}\left[C e^{\mathrm{i} \theta}\left(E-E_{\mathrm{ex}}+\mathrm{i} \Gamma\right)^{-m}\right],
$$

where $C, \theta, E_{\mathrm{ex}}$, and $\Gamma$ are the amplitude, phase, energy, and broadening parameter of the spectral line, respectively. Parameter values of $m=1.5$ or $m=3$ were used in the calculations. In the case of $m=1.5$, expression (1) represents quite well the Gaussian profile of a state-filling modulated line. ${ }^{24}$ While using the index $m=3$ yields the first derivative of a Gaussian absorption profile, ${ }^{27}$ which is acceptable for quantum systems with inhomogeneously broadened energy levels. The moduli of individual PR resonances (individual optical transitions) were evaluated from fitting the PR spectra according to ${ }^{16}$

$$
|\Delta \rho(E)|=\frac{C}{\left[\left(E-E_{\mathrm{ex}}\right)^{2}+\Gamma^{2}\right]^{m / 2}} .
$$

It should be noted that peak positions in the PR modulus spectra correspond to the optical transition energies, whilst their heights are proportional to the intensity (probability) of the transitions.

\section{Temperature-dependent photoreflectance spectra}

From the discussion in Sec. III A, it follows that the BSR effect may complicate the modulated reflectance spectra for multilayer QD structures. Nevertheless, in some cases this effect can be exploited in order to enhance weak signals from QD-related optical transitions. In this study, however, to avoid the influence of BSR effects, only the temperature- dependent PR spectra of DWELL samples with rough backsurface were examined.

The PR spectra for DWELL structure (with sanded back-surface) measured at different temperatures in the photon energy region of InAs QD and InGaAs bi-QW optical transitions are presented in Fig. 4. The blue-shift of all the main PR features expected as the temperature is reduced can be attributed to the bandgap change of the QD/QW materials. One can also see a temperature-dependent variation of the relative intensity and line shape of the QD and QW features that is quite unusual. The most pronounced changes occur for the QD-related features. When the temperature is decreased from $300 \mathrm{~K}$ to about $150 \mathrm{~K}$, the line-shape of the QD spectral PR features transforms, while their intensity significantly increases, as clearly seen in Fig. 4.

By comparing the temperature-dependent PR and PL spectra, we found that a progressive increase in the QD PR intensity correlates with the emergence of the QD PL emission peak. The impact of temperature on the integrated PL intensity and PR signal amplitude (peak-to-peak) for the QD GS transitions in DWELL structures is depicted in Fig. 5. By lowering the temperature from 300 to $150 \mathrm{~K}$, the PR signal experiences an enhancement nearly identical to that of the PL signal intensity. The Arrhenius-type behavior observed in Fig. 5 implies that the PR intensity is linked to the carrier capture/population probability within the QDs, whereas the temperature variation of the PR signal can be related to the escape efficiency of the photogenerated carriers from InAs QDs to InGaAs QW and nonradiative recombination centers. Indeed, the derived activation energy of $\sim 320 \mathrm{meV}$ is close to the energy difference $\Delta E=E_{\mathrm{QW}}^{\mathrm{GS}}-E_{\mathrm{QD}}^{\mathrm{GS}}$ (total barrier height). This thermal quenching effect in this temperature range was also discussed in our previous work ${ }^{28}$ and has

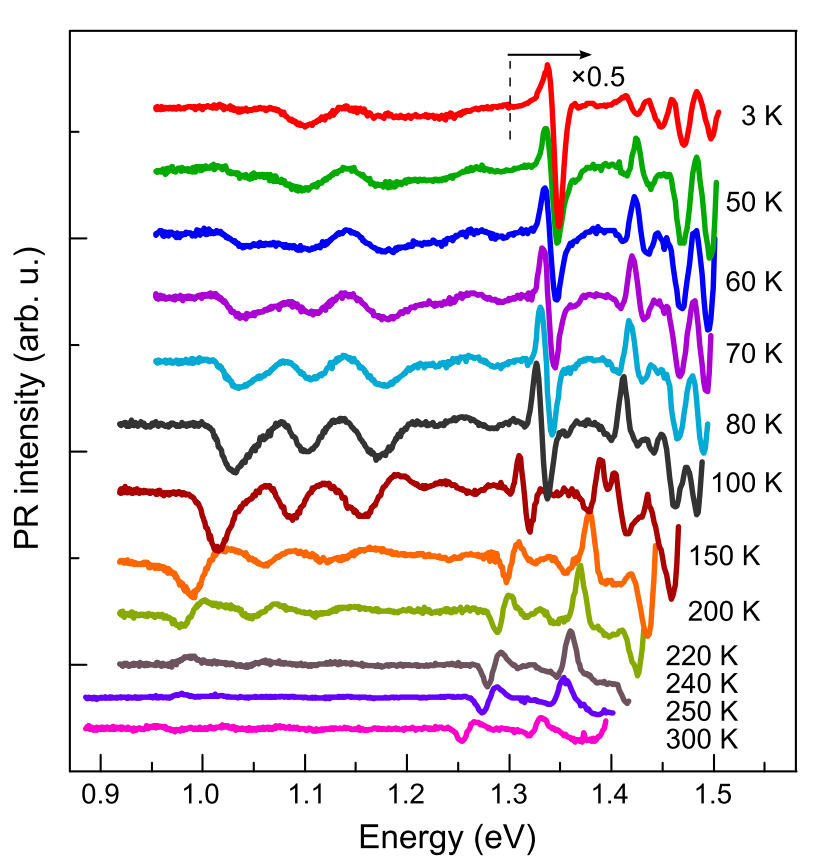

FIG. 4. Temperature-dependent PR spectra for the DWELL structure with sanded back-surface, showing the evolution of optical transitions originating within the InAs QDs and InGaAs bi-QW. 


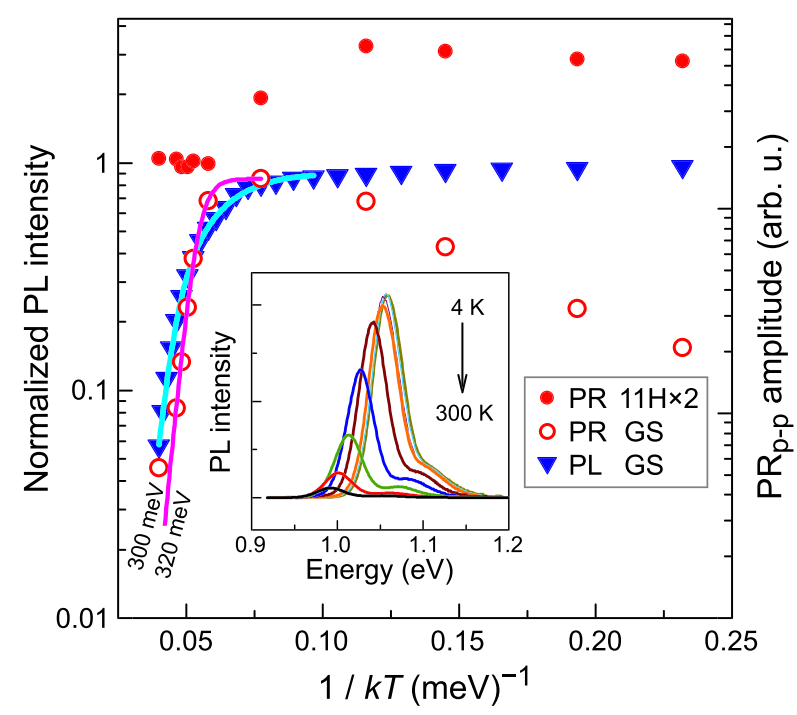

FIG. 5. Arrhenius plots of the integrated PL intensity along with the PR peak-to-peak signal amplitude for the GS transitions in the QDs. Temperature dependence of the heavy-hole related interband transition $(11 \mathrm{H}) \mathrm{PR}$ intensity (doubled) for the InGaAs QW is given for comparison. Symbols correspond to the experimental data and straight lines to the simulation. Inset: PL spectra as a function of temperature.

been accounted for by carrier thermal escape from QD to QW states with an activation energy of $300 \mathrm{meV}$.

The enhancement of PR signal observed whilst reducing the sample temperature down to $150 \mathrm{~K}$ (see Fig. 4) can thus be associated with the capture of photoexcited carriers into QDs leading to GS filling effects and/or screening of local internal electric field. ${ }^{29}$ With further decrease of the temperature down to $3 \mathrm{~K}$, the QD GS signal intensity diminished gradually, probably due to the state-filling effect. In contrast, the QW related PR intensity increased with reduction in the temperature. The origin of the lineshape variation of the PR spectra is still under investigation; however, it is plausible that it is related to the variation of photomodulation mechanism with temperature: from photomodulation of the built-in electric field (high $T$ ) to an intrinsic QD mechanisms (low $T$ ). A more detailed analysis of the PR spectra shows that, at high temperatures, the lineshape is dominated by the QCSE. ${ }^{10}$ Whereas at low temperatures, the PR lineshape indicates the presence of state-filling mechanism, ${ }^{23}$ and this is supported by the trend of suppressing the QD transitions. Alongside this modulation mechanism, the PR spectra contain useful information about optical transitions in DWELL structures, and this is of the main interest in this study.

To determine transition energies and broadening parameters, curve fitting of the PR spectra was performed using the functional form of Eq. (1) and is plotted in Fig. 6 for several representative temperatures. The analysis of the PR spectra revealed the GS and four ES transitions (see PR modulus lines in Fig. 6); this points to a high uniformity of the QDs under study. Also, in the low temperature region, an additional feature is resolved at high-energy close to the lowest QW resonance $11 \mathrm{H}$. We believe that this originates from a "crossed" optical transitions involving a QD state and a QW state. ${ }^{30}$

The temperature dependence of GS and ES transition energies for QD ensembles obtained from the PR spectra is

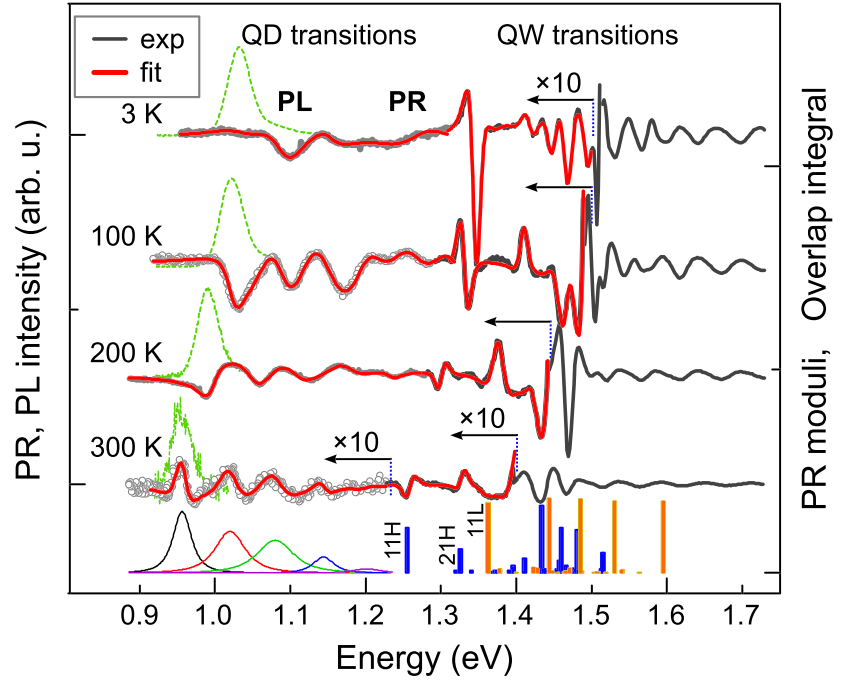

FIG. 6. Experimental PR and PL spectra for several representative temperatures. The thick solid lines are least-square fits of PR, whilst thin lines (bottom) are the moduli of QD PR resonances at $300 \mathrm{~K}$. The positions and amplitudes of vertical bars denote calculated optical transition energies and intensities at $300 \mathrm{~K}$.

presented in Fig. 7, with some of the corresponding PL peak energies (if observed) plotted for comparison. The obtained interband transition energies are in a reasonable agreement with the PL peak energies. As a result, the Stokes shift between the emission-like PL and the absorption-like PR spectra seems not to be significant for these samples.

All the plots of the variation of transition energy with temperature are nearly parallel, with an almost equidistant spacing of $\sim 60 \mathrm{meV}$. This indicates that electrons are confined in a parabolic-like potential within the lens-shaped QDs. ${ }^{31,32}$ It can be noted in Fig. 7 that the experimentally observed temperature dependence of the optical transition energies (symbols) fit fairly well with the semi-empirical Varshni expression ${ }^{33}$ (lines)

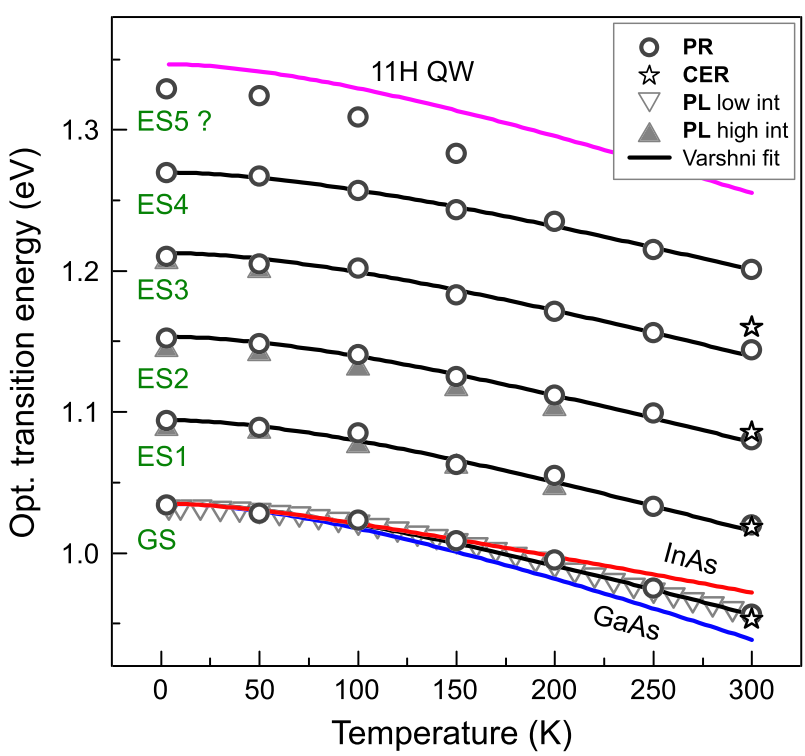

FIG. 7. The energies of QD optical transitions as a function of temperature. The symbols are experimental data, whereas the solid lines are fitted using the Varshni formula (3). 
TABLE I. Varshni fitting parameters.

\begin{tabular}{lccr}
\hline \hline Optical Transitions & $E_{0}(\mathrm{eV})$ & $\alpha(\mathrm{meV} / \mathrm{K})$ & $\beta(\mathrm{K})$ \\
\hline GS & 1.035 & 0.429 & 190 \\
ES1 & 1.094 & 0.429 & 190 \\
ES2 & 1.153 & 0.412 & 195 \\
ES3 & 1.213 & 0.404 & 196 \\
ES4 & 1.269 & 0.389 & 207 \\
$11 \mathrm{H}$ QW & 1.347 & 4.980 & 191 \\
InAs & 35 & 0.276 & 93 \\
$\mathrm{GaAs}^{35}$ & 0.415 & 0.540 & 204 \\
\hline \hline
\end{tabular}

$$
E(T)=E_{0}-\frac{\alpha T^{2}}{T+\beta} .
$$

The fitting parameters of $E_{0}, \alpha$, and $\beta$ were estimated from the temperature variation of the PL and PR features corresponding to InAs QD-related GS and ES transitions, along with InGaAs bi-QW main $(11 \mathrm{H})$ transition, and are listed in Table I. In addition, the corresponding parameters of the energy bandgap variation with temperature in the bulk InAs and $\mathrm{GaAs}$ crystals are presented for comparison.

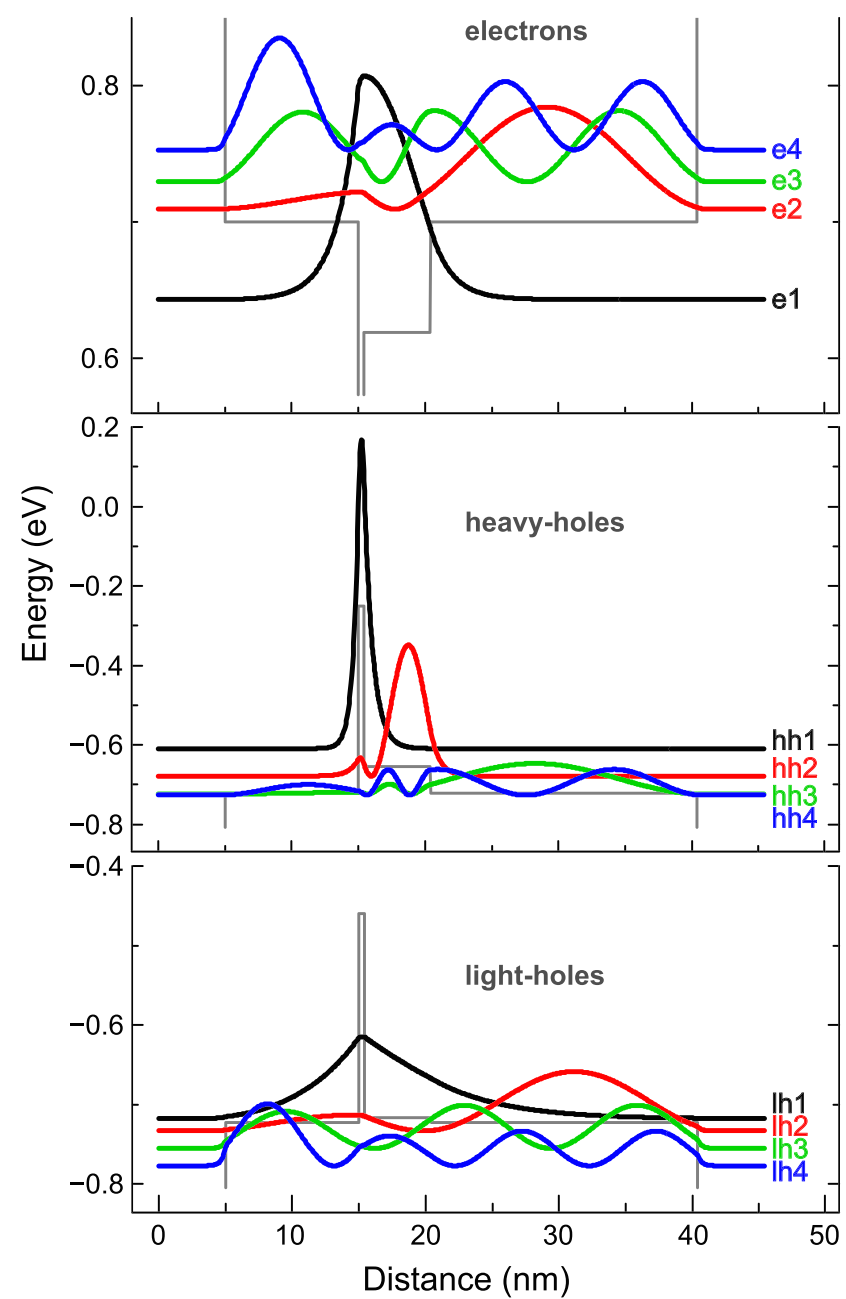

FIG. 8. The calculated potential profiles, energy levels, and squared moduli of wavefunctions for electrons, heavy-, and light-holes in InAs/InGaAs/ GaAs/AlAs double QW. The calculations were performed under flat band conditions, $F=0$.

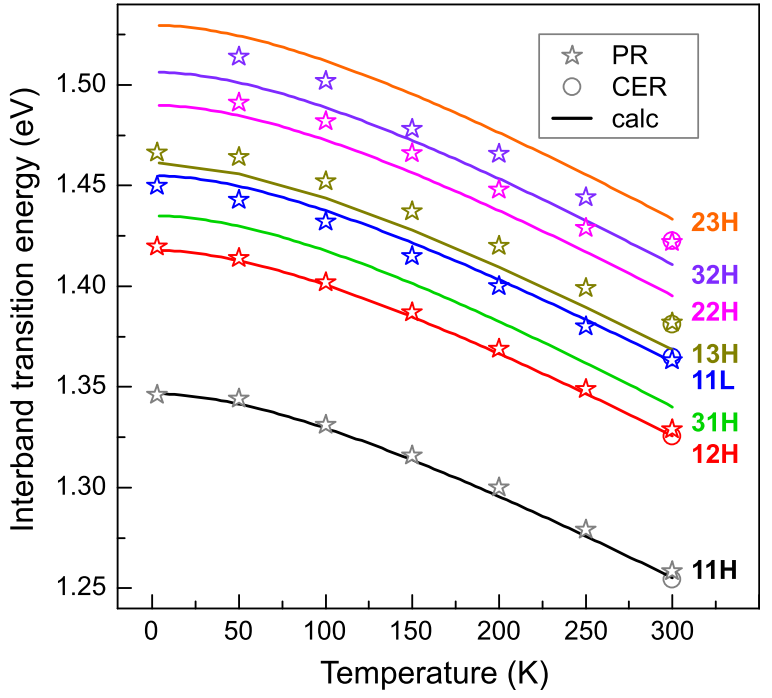

FIG. 9. Calculated (solid lines) and experimental (symbols) transition energies for InAs/InGaAs/GaAs/AlAs double QWs as a function of temperature.

The parameters for the QD ensembles obtained by the fitting are somewhat larger than those reported for bulk InAs. This observation indicates that the QD composition of the studied DWELL is partially changed due to Ga/In interdiffusion. $^{34}$ In fact, for high-uniformity QD structures the main reason for spectral shifts with temperature is the temperature variation of the material bandgaps. Therefore, it is proposed that changes of QD size and composition are a result of the decomposition of the InGaAs capping layer. ${ }^{35}$ To estimate the actual In content in the $\operatorname{In}_{x} \mathrm{Ga}_{1-x}$ As cap layer, the temperature dependence of QW-related transitions (seen in the PR spectra of Fig. 6) was examined in more detail. To identify the observed spectral features, calculations of energy levels and interband transition energies for the complex QW structure consisting of InAs WL and InGaAs cap layer embedded in a GaAs/AlAs well were carried out using an effective mass approximation by means of the nextnano ${ }^{3}$ software package. ${ }^{36}$ The relevant material parameters were taken from Ref. 37.

The calculated potential profiles, energy levels, and squared moduli of wavefunctions for electrons, heavy-, and light-holes in InAs/InGaAs/GaAs/AlAs double QW structures under zero electric field conditions are presented in Fig. 8. The breaking of wavefunction symmetry for the asymmetric structure is clearly seen. Consequently, several types of interband transitions from the initial $m$ to the final $n$ states can be observed in PR spectra: (i) the transitions with $m=n$, such as $11 \mathrm{H}$ or $22 \mathrm{H}$, which are allowed in simple symmetric QWs, (ii) the transitions with $m \neq n(12 \mathrm{H}, 21 \mathrm{H}$, etc.), which are normally forbidden in symmetric QWs but due to the asymmetryinduced relaxation of selection rules now become allowed even at zero electric field. Here, the notation $m n \mathrm{H}(\mathrm{L})$ is used for transitions between the $m$ th electron and the $n$th heavy(light-) hole subbands. It is important to emphasize that optical transitions between the local states (confined in a deep narrow $\mathrm{QW}$ ) and global ones (confined in a wide $\mathrm{QW}$ ) account for the specific features of DWELL photodetectors, such as a bias-tunable spectral response. ${ }^{38}$ When associating certain PR 
features with various optical transitions, it is essential to consider their oscillator strengths (transition probabilities), which are proportional to the squares of the overlap integrals between the electron and hole wavefunctions.

Vertical bars in Fig. 6 (bottom) denote calculated energies and intensities of interband transitions within InGaAs biQWs at room temperature. As seen, the distinct spectral features which are more pronounced at low temperature occur near predicted transitions. However, some calculated transitions are of low intensity, or overlapping in energy, and thus cannot be resolved/detected. The optical transition energies as a function of temperature for calculated and experimental data are presented in Fig. 9. The agreement between the experimental points and the calculated curves is quantitatively good. A comparison of experimental data and simulations yields an actual value of $x=0.13$ for the $\mathrm{In}_{\mathrm{x}} \mathrm{Ga}_{1-x}$ As capping layer. This is consistent with the degree of the strain-driven partial decomposition of the $\mathrm{In}_{0.15} \mathrm{Ga}_{0.85}$ As cap layer obtained from optical investigations reported previously. ${ }^{35}$

\section{CONCLUSIONS}

Temperature-dependent (3-300 K) PR spectroscopy was used to study optical transitions within dots-in-a-well photodetector structures. Cumulative analysis of PR, phototransmittance, and contactless-electroreflectance spectra revealed the influence of back-surface reflections on the line shape of photomodulated reflectance. The interband optical transition energies involving ground- and excited-states for InAs QDs and InGaAs quantum well were established. An Arrhenius analysis gave a $\sim 320 \mathrm{meV}$ activation energy at high temperatures, suggesting that signal intensity quenching is governed by exciton escape from dot-to-well bound-states. It was found that the temperature dependence of QD-related optical transitions follows the Varshni relation with parameters larger than reported for a bulk InAs, suggesting a straindriven partial decomposition of the InGaAs cap layer. The complex nature of the photomodulation mechanism for multilayer QD photodetector structures is shown to be associated with the QD doping and internal electric fields.

\section{ACKNOWLEDGMENTS}

The current research was funded by a grant (No. MIP071/2012) from the Research Council of Lithuania. We also acknowledge support from the EPSRC (UK) and ERC programme "TOSCA".

${ }^{1}$ A. Stintz, G. T. Liu, H. Li, L. F. Lester, and K. J. Malloy, IEEE Photon. Technol. Lett. 12(6), 591-593 (2000).

${ }^{2}$ M. T. Crowley, N. A. Naderi, H. Su, F. Grillot, and L. F. Lester, in Advances in Semiconductor Lasers, Semiconductors and Semimetals Vol. 86, edited by J. J. Coleman, A. C. Bryce, and C. Jagadish (Academic Press, San Diego, 2012), pp. 371-417.

${ }^{3}$ A. V. Barve, S. J. Lee, S. K. Noh, and S. Krishna, Laser Photonics Rev. 4(6), 738-750 (2010).

${ }^{4}$ A. V. Barve and S. Krishna, in Advances in Infrared Photodetectors, Semiconductors and Semimetals Vol. 84, edited by S. D. Gunapala, D. R. Rhiger, and C. Jagadish (Academic Press, San Diego, 2011), pp. 153-193.
${ }^{5}$ A. Barve, J. Shao, Y. D. Sharma, T. E. Vandervelde, K. Sankalp, S. J. Lee, S. K. Noh, and S. Krishna, IEEE J. Quantum Electron. 46(7), 1105-1114 (2010).

${ }^{6}$ Y.-F. Lao, S. Wolde, A. G. U. Perera, Y. H. Zhang, T. M. Wang, J. O. Kim, T. Schuler-Sandy, Z.-B. Tian, and S. S. Krishna, Appl. Phys. Lett. 104, 171113 (2014).

${ }^{7}$ H. W. Shin, J.-W. Choe, S. J. Lee, S. K. Noh, J. O. Kim, and K.-S. Lee, Curr. Appl. Phys. 14(5), 721-724 (2014).

${ }^{8}$ B. Čechavičius, J. Kavaliauskas, G. Krivaite, D. Seliuta, G. Valušis, M. P. Halsall, M. J. Steer, and P. Harrison, J. Appl. Phys. 98(2), 023508 (2005).

${ }^{9}$ M. Motyka, R. Kudrawiec, G. Sęk, J. Misiewicz, I. L. Krestnikov, S. Mikhrin, and A. Kovsh, Semicond. Sci. Technol. 21, 1402 (2006).

${ }^{10}$ M. Motyka, G. Sęk, R. Kudrawiec, J. Misiewicz, L. H. Li, and A. Fiore, J. Appl. Phys. 100, 073502 (2006).

${ }^{11}$ T. K. Sharma, T. J. C. Hosea, S. J. Sweeney, and X. Tang, J. Appl. Phys. 104, 083109 (2008).

${ }^{12}$ M. Motyka, R. Kudrawiec, G. Sęk, J. Misiewicz, D. Bisping, B. Marquardt, A. Forchel, and M. Fischer, Appl. Phys. Lett. 90, 221112 (2007).

${ }^{13}$ R. Nedzinskas, B. Čechavičius, J. Kavaliauskas, V. Karpus, G. Krivaitė, V. Tamošiūnas, G. Valušis, F. F. Schrey, K. Unterrainer, and G. Strasser, Acta Phys. Pol. A 113, 975 (2008), available at http://przyrbwn.icm.edu. pl/APP/PDF/113/a113z342.pdf.

${ }^{14}$ R. Nedzinskas, B. Čechavičius, J. Kavaliauskas, V. Karpus, D. Seliuta, V. Tamošiūnas, G. Valušis, G. Fasching, K. Unterrainer, and G. Strasser, J. Appl. Phys. 106, 064308 (2009).

${ }^{15}$ V. K. Dixit, S. K. Khamari, C. Tyagi, S. D. Singh, S. Porwal, R. Kumar, C. Mukherjee, P. Mondal, A. K. Srivastava, T. K. Sharma, and S. M. Oak, J. Phys. D: Appl. Phys. 45, 365104 (2012).

${ }^{16}$ J. Misiewicz and R. Kudrawiec, Opto-Electron. Rev. 20(2), 101-119 (2012).

${ }^{17}$ M. Motyka, K. Ryczko, M. Dyksik, G. Sęk, J. Misiewicz, R. Weih, M. Dallner, S. Höfling, and M. Kamp, Appl. Phys. Lett. 117, 084312 (2015).

${ }^{18}$ W. M. Theis, G. D. Sanders, C. E. Leak, K. K. Bajaj, and H. Morkoc, Phys. Rev. B 37, 3042 (1988).

${ }^{19}$ R. L. Tober and J. D. Bruno, J. Appl. Phys. 68, 6388 (1990).

${ }^{20}$ H. Roppischer, N. Stein, U. Behn, and A. B. Novikov, J. Appl. Phys. 76, 4340 (1994).

${ }^{21}$ A. J. Shields and P. C. Klipstein, Phys. Rev. B 43, 9118 (1991).

${ }^{22}$ Zh. M. Wang, Y. I. Mazur, Sh. Seydmohamadi, G. J. Salamo, and H. Kissel, Appl. Phys. Lett. 87, 213105 (2005).

${ }^{23}$ T. M. Hsu, W.-H. Chang, C. Y. Lai, N. T. Yeh, and J.-I. Chyi, J. Appl. Phys. 91, 4399 (2002)

${ }^{24}$ W.-H. Chang, T. M. Hsu, N. T. Yeh, and J.-I. Chyi, Phys. Rev. B 62, 13040 (2000).

${ }^{25}$ E. W. Bogaart, J. E. M. Haverkort, T. Mano, T. van Lippen, R. Nötzel, and J. H. Wolter, Phys. Rev. B 72, 195301 (2005).

${ }^{26}$ D. E. Aspnes, Surf. Sci. 37, 418-442 (1973).

${ }^{27}$ B. V. Shanabrook, O. J. Glembocki, and W. T. Beard, Phys. Rev. B 35, 2540 (1987).

${ }^{28}$ R. Nedzinskas, B. Čechavičius, A. Rimkus, J. Kavaliauskas, G. Valušis, L. H. Li, and E. H. Linfield, Lith. J. Phys. 54, 54-57 (2014).

${ }^{29}$ V. Mitin, A. Antipov, A. Sergeev, N. Vagidov, D. Eason, and G. Strasser, Nanoscale Res. Lett. 6, 21 (2011).

${ }^{30}$ G. Visimberga, G. Rainò, A. Salhi, V. Tasco, M. T. Todaro, L. Martiradonna, M. De Giorgi, A. Passaseo, R. Cingolani, and M. De Vittorio, Appl. Phys. Lett. 93, 151112 (2008).

${ }^{31}$ S. Raymond, S. Fafard, P. J. Poole, A. Wojs, P. Hawrylak, S. Charbonneau, D. Leonard, R. Leon, P. M. Petroff, and J. L. Merz, Phys. Rev. B 54, 11548 (1996).

${ }^{32}$ R. Heitz, O. Stier, I. Mukhametzhanov, A. Madhukar, and D. Bimberg, Phys. Rev. B 62, 11017 (2000).

${ }^{33}$ Y. P. Varshni, Physica 34(1), 149-154 (1967).

${ }^{34}$ F. Ferdos, S. Wang, Y. Wei, M. Sadeghi, Q. Zhao, and A. Larsson, J. Cryst. Growth 251(1-4), 145-149 (2003).

${ }^{35}$ F. Guffarth, R. Heitz, A. Schliwa, O. Stier, N. N. Ledentsov, A. R. Kovsh, V. M. Ustinov, and D. Bimberg, Phys. Rev. B 64, 085305 (2001).

${ }^{36}$ See http://www.nextnano.de/nextnano3 for nextnano ${ }^{3}$, next generation 3D nanodevice simulator.

${ }^{37}$ I. Vurgaftman, J. R. Meyer, and L. R. Ram-Mohan, J. Appl. Phys. 89, 5815 (2001).

${ }^{38}$ P. Aivaliotis, N. Vukmirović, E. A. Zibik, J. W. Cockburn, D. Indjin, P. Harrison, C. Groves, J. P. R. David, M. Hopkinson, and L. R. Wilson, J. Phys. D: Appl. Phys. 40, 5537 (2007). 